\title{
Envelhecer@Saudável: um material educacional digital voltado ao público idoso
}

\author{
Maria Lúcia Kroeff Barbosa ${ }^{1}$, Cleonice Silvana Rodrigues Hahn², \\ Deyse Cristina Frizzo Sampaio ${ }^{3}$, Luziana Quadros da Rosa ${ }^{4}$, \\ Sílvio César Cazella ${ }^{1,5}$, Letícia Rocha Machado ${ }^{1}$ \\ ${ }^{1}$ Programa de Pós-Graduação em Informática na Educação - UFRGS \\ ${ }^{2}$ Programa de Pós-Graduação em TICs na Educação - FURG \\ ${ }^{3}$ Núcleo de Tecnologia Digital Aplicada à Educação - UFRGS \\ ${ }^{4}$ Programa de Pós-Graduação em Tecnologias da Informação e Comunicação - UFSC \\ ${ }^{5}$ Programa de Pós-Graduação em Ensino na Saúde - UFCSPA \\ malukroeff@yahoo.com.br, cleonicehahn@gmail.com, deysefrizzo@gmail.com, \\ luziquadros@hotmail.com, silvio.ufcspa@gmail.com, leticiarmachado@gmail.com \\ Resumo. Este artigo tem como objetivo apresentar o planejamento e a \\ implementação de um material educacional digital (MED) denominado \\ Envelhecer@Saudável, o qual busca promover o conhecimento e mudanças de \\ hábitos para melhores condições de saúde e de bem-estar do idoso. A \\ metodologia adotada engloba quatro etapas de desenvolvimento: organização \\ da equipe; planejamento; escolha do formato e modelo; inclusão dos recursos \\ multimídias. $O$ MED foi adaptado as especificidades do público-alvo e teve \\ como foco formatos e tendências inovadoras, adaptáveis à interação e às \\ novas possibilidades para aquisição de conhecimentos e, também, uma maior \\ inclusão digital do idoso.
}

Palavras-Chave: material educacional digital, educação de idosos, inclusão digital, envelhecimento

\section{Aging@Healthy: a digital educational material aimed at the elderly population}

\begin{abstract}
This paper aims to present the planning and implementation of a digital educational material (MED) named Aging@Healthy, which seeks to promote knowledge and changes in health habits through awareness to better health and well-being of the elderly. Initially, the methodology has four stages of development: team organization; planning; choice of format and style; inclusion of multimedia resources. The MED was adapted the specifics of the target audience and sought to have focused formats and innovative trends, adaptive interaction and new opportunities to acquire knowledge and also greater digital inclusion of the elderly.
\end{abstract}

Keywords: digital educational material, elderly education, digital inclusion, aging 


\section{Introdução}

No Brasil, a população idosa cresce em proporções geométricas trazendo inúmeras demandas, entre elas a inserção na era digital por meio da utilização de recursos tecnológicos com alternativas para a inclusão e a acessibilidade no mundo contemporâneo. No entanto, é fundamental considerar peculiaridades próprias dessa etapa da vida, como a sua história, suas condições cognitivas, físicas e emocionais. A expectativa de vida atual aponta que a mortalidade brasileira declinou e a perspectiva é que, se forem mantidas as tendências registradas das últimas duas décadas, até 2030 as mulheres viverão em média 85,3 anos, e os homens, 78,1 anos [Portal Brasil, 2014]. Este cenário longevo apresenta inúmeras mudanças e quebra de paradigmas, pois boa parcela da população idosa busca estar ativa e interessada em aprimorar ou desenvolver novas competências, procurando tanto a inserção social quanto a inclusão digital.

Segundo Rodrigues e Pereira [2016], o conceito de velhice tem passado por transformações devido ao novo papel do idoso na sociedade moderna. Assim, novas perspectivas estão surgindo, possibilitando maneiras diversas de vivenciar essa etapa da vida, como uma fase de valorização e compreensão da vivência e como algo ainda proveitoso e valioso. Ou seja, os idosos de hoje estão enfrentando novos desafios e afastando-se cada vez mais dos idosos do passado. A internet e as tecnologias digitais vêm oportunizando, portanto, uma nova forma de obter informação, estimular o raciocínio, proporcionar momentos de lazer e socialização, favorecendo a inclusão digital [Nunes 2002 apud Zimermman, Mozzaquatro e Cunha, 2013].

Conforme Passerino e Pasqualotti [2006], Doll, Cachioni e Machado [2016], a inclusão digital é vista como uma forma de inclusão social, porque através das tecnologias digitais é possível ao idoso uma participação mais ativa na sociedade, promovendo diferentes formas de acesso à informação e um melhor desenvolvimento social, cognitivo e afetivo. Com isso, percebe-se que a inclusão digital de idosos possui enorme relevância e representa um grande desafio na atualidade. Ou seja, importante cada vez mais investigar e discutir sobre possíveis abordagens educacionais através do uso das tecnologias digitais.

Kachar [2010] aponta que a abordagem educacional que pode ser utilizada com os idosos, referente ao ensino permeado pela tecnologia, deve ser bem específica, requer compreensão e necessita de uma prática pedagógica que considere as características físicas, psicológicas e sociais desta faixa etária. A partir deste cenário, observa-se que o uso de materiais educacionais digitais pode se tornar uma excelente proposta de estratégia pedagógica para a inclusão digital de idosos. Segundo Torrezzan e Behar [2016], o termo Material Educacional Digital (MED) está relacionado a todo o material voltado à aprendizagem e que utiliza um ou mais recursos digitais na sua elaboração. Assim, é pertinente o desenvolvimento de materiais educacionais digitais para idosos no intuito de atender este público tanto em relação às suas necessidades cognitivas e motoras, como também seu contexto social e vivências [Grande, 2016].

Com base no descrito acima, o objetivo principal deste artigo é apresentar o planejamento e a implementação do MED denominado Envelhecer@Saudável. Este MED visa favorecer o processo de ensino e aprendizagem, assumindo um papel motivador para promover conhecimento e mudança de hábitos com os idosos por meio da conscientização para melhores condições de saúde e de bem-estar. 
Sendo assim, este artigo está organizado de maneira a apresentar uma contextualização sobre o envelhecimento e as tecnologias digitais, bem como o referencial teórico que embasou o planejamento do MED e as etapas desenvolvidas para sua implementação. $\mathrm{Na}$ seção 2 será feita uma análise que permeia sobre $\mathrm{o}$ envelhecimento, as tecnologias digitais e o bem-estar do idoso. A seção 3 descreve sobre os materiais educacionais digitais e o seu uso com a população de mais idade, apresentando alguns trabalhos relacionados. Na seção 4, será apresentado o referencial teórico sobre possíveis métodos utilizados para construção de MEDs. A seção 5 traz a implementação do Envelhecer@Saudável, e analisa aspectos relevantes à sua utilização frente a um público de mais idade. Na seção 6 , as considerações finais.

\section{Envelhecimento, tecnologias digitais e bem-estar}

Nas últimas décadas, o conceito de envelhecimento vem sendo estudado com mais profundidade, principalmente devido ao aumento do público idoso. Para compreender as modificações e o complexo processo de envelhecer é necessário conceituar diferentes termos que estão relacionados. Segundo Kachar [2003], o envelhecimento normal é considerado como senescência. Já a senilidade apresenta uma ou mais patologias associadas a fatores ambientais e/ou genéticos e é caracterizada por disfunções decorrentes de doenças degenerativas da velhice. Tende-se, assim, por associar o envelhecimento a aspectos negativos, tanto pela população de um modo geral, quanto pelos próprios idosos. Porém, segundo a autora, o perfil do idoso do século XXI mudou, uma vez que deixou de ser uma pessoa recolhida em seu aposento, vivendo de lembranças do passado, para uma pessoa ativa capaz de produzir e intervir nas mudanças sociais e políticas. Diferentemente dos sujeitos que já nasceram durante o surgimento e ascensão das tecnologias digitais, a geração de mais idade normalmente tem uma convivência conflituosa em meio às complexas e rápidas mudanças tecnológicas. Sendo assim, torna-se importante investigar quais as abordagens adequadas para introduzir os idosos no universo da tecnologia digital e construir estratégias e materiais educacionais, a fim de prepará-los adequadamente para o domínio dos recursos computacionais.

De acordo com a Organização Mundial da Saúde (OMS), e conforme Machado e Behar [2015] descrevem, uma das formas de propiciar a qualidade de vida, é a participação em atividades que favoreçam o bem-estar, entre elas a educação. As autoras apontam para o fato de que, nas últimas décadas, as tecnologias digitais estão se tornando uma fonte de entretenimento social e cultural, além de um local de informações e comunicação da população. Este panorama possibilitou a discussão sobre o uso das tecnologias digitais pelos idosos. Nessa perspectiva, a seguir serão esclarecidos alguns aspectos importantes sobre materiais educacionais digitais (MEDs), de acordo com as necessidades do usuário.

\section{Material Educacional Digital e idosos}

Embora os avanços das tecnologias digitais tenham possibilitado inúmeros benefícios ao diminuir distâncias, ao tornar as informações mais acessíveis e a vida mais fácil, provocou também a exclusão de alguns grupos sociais, sobretudo dos idosos, frente as limitações como da visão, da audição, do controle motor e da cognição. Para o enfrentamento dessas alterações funcionais, inerentes a todo ser humano com o passar do tempo, faz-se necessário a adoção de medidas de modo a favorecer a usabilidade das 
tecnologias digitais por esta parcela da população. Nesse contexto, os MEDs possibilitam a criação de estratégias como apoio para o ensino e aprendizagem para o público idoso ao considerar sua história de vida, seu cotidiano, seus interesses, sua linguagem, suas necessidades, bem como, suas alterações funcionais, que segundo Tavares e Souza [2012], parte da população idosa apresenta no decorrer de sua vida dificuldades de ordem cognitiva, motora, talvez financeira, que podem ser fatores limitantes ao acesso às novas tecnologias. .

Torrezzan e Behar [2016] conceituam o termo material educacional digital como todo o material voltado à aprendizagem e que utiliza um ou mais recursos digitais na sua elaboração. As autoras colocam que, inicialmente, os MEDs eram praticamente elaborados a partir da simples digitalização de materiais educacionais analógicos. Atualmente, existe uma maior preocupação em pensar novas estratégias pedagógicas que se relacionem, de maneira harmônica, aos recursos digitais e que sejam centradas na aprendizagem do aluno. Esse fato torna necessária a integração de métodos distintos e tutoriais que tratem individualmente dessas questões, fornecendo complexidade à prática da equipe desenvolvedora, muitas vezes composta pelo professor (conteudista), designer e programador. Como exemplos de MEDs citam as páginas web, blogs, softwares educacionais, aplicativos, objetos de aprendizagem (OA), entre outros.

\subsection{Trabalhos Relacionados}

O objeto de aprendizagem "Iniciação à Internet" (INICIANET) tem por objetivo introduzir o público idoso nos conceitos básicos da internet, assim como as suas ferramentas [Machado e Behar, 2010]. Considera como aspectos essenciais a maior liberdade do aluno para escolher o que deseja aprofundar, além da liberdade também na mediação do professor. É composto por seis tópicos (internet, comunidades virtuais, email, pesquisa na internet, blogs e flogs, wikis) que contemplam, em cada um, atividades interativas além de recursos multimídias como apresentações, animações, vídeos, áudios e hipertexto.

O "ConReDis - Construção de Redes Sociais Digitais para Idosos" aborda os conceitos e recursos da internet, porém com foco nas redes sociais virtuais. O layout foi estruturado de forma que o conteúdo disposto seja de fácil acesso e compreensão, considerando as cores, as fontes ideais para pessoas que já possuem dificuldade de visão, botões padrões e organização do conteúdo de forma que o idoso, que não possui grande vivência virtual, não se perca entre muitos links e imagens aleatórias [Pinto et al., 2014].

Outro material digital que vale citar é o "DIMOS - Dispositivos Móveis e Seniors: uso e aplicação" [Fonseca et al., 2015], voltado para a o público idoso que possui interesse em aprender a manusear um dispositivo móvel, especificamente o tablet. No objeto, são abordados conceitos e atividades, no formato de desafios, referentes ao uso dos recursos digitais móveis. A equipe desenvolvedora interdisciplinar envolveu pesquisadores, profissionais e alunos da pedagogia, gerontologia e design.

Nesse sentido, percebe-se que um material didático voltado ao público idoso necessita utilizar recursos que sejam atrativos e que facilitem o seu acesso oferecendo segurança, e sobretudo, possa minimizar as dificuldades de algumas alterações funcionais decorrentes da idade estimulando a sua utilização pelo usuário, de modo a alcançar os objetivos propostos da sua construção ao promover atividades participativas e interativas, bem como, temas que contribuam para a melhoria da sua qualidade de vida. 
Conforme Macedo e Pereira [2009], faz-se necessário buscar estratégias para que o MED beneficie o usuário idoso a perceber, compreender, navegar e interagir com as informações disponíveis facilitando, dessa forma, sua aprendizagem e sua inclusão no mundo virtual e globalizado. Com base nesses aspectos, a próxima seção trata da metodologia utilizada para a construção do Envelhecer@Saudável.

\section{Referencial Teórico}

A construção e viabilidade dos materiais educacionais digitais dependem de fatores previamente estabelecidos. Existem muitos métodos sendo adotados para $\mathrm{o}$ desenvolvimento de MEDs. Amante e Morgado [2001] sugerem as seguintes etapas:

a) Concepção do Projeto: o que, para quem e de que forma desenvolver.

b) Planificação: criar o storyboard (maquete), selecionar materiais e conteúdos, construir esquemas e protótipos, design da interface.

c) Implementação: construção do planejamento e da planificação.

d) Avaliação: validação.

Já Torrezan [2009], baseada nos parâmetros do design pedagógico, considera alguns fatores necessários na construção de MEDs, ressaltando-se que esses fatores não agem de maneira totalmente isolada e um geralmente atua sobre o outro:

$\checkmark$ Fatores Gráficos: compostos pela estética e design, e devem apoiar o usuário na construção do conhecimento.

$\checkmark$ Fatores Técnicos: relacionados às questões de ergonomia e programação informática do MED, apoiando a trajetória autônoma e não-linear do usuário.

$\checkmark$ Fatores Pedagógicos: baseados nas teorias construtivistas de Piaget [1974], buscam relacionar aspectos que dizem respeito ao perfil do usuário, à elaboração do conteúdo abordado e ao planejamento das interações e interatividades possibilitadas por meio da relação usuário-MED.

Outro método utilizado para construção desses materiais digitais e baseado no design pedagógico, é abordado por Passos [2011] e composto por cinco etapas:

1. Compreensão: compreensão do projeto e levantamento de informações sobre o tema, objetivos pedagógicos e perfil dos usuários, entre outros.

2. Preparação: descrição dos elementos e funcionalidades necessárias, definição da equipe, seleção dos recursos digitais, busca do conteúdo a ser abordado.

3. Experimentação: organização do conteúdo conforme a proposta interativa, envolvendo o design de interação e desenho dos fluxos de tarefa.

4. Elaboração: realização do projeto gráfico, da definição da hierarquia da informação, além da modularização (composição dos elementos gráficos) e agrupamento do conteúdo.

5. Apresentação: elaboração do design, definição dos atributos gráficos, montagem da apresentação de textos e imagens.

A seguir, é apresentado o material educacional digital Envelhecer@Saudável, bem como sua composição.

\section{MED Envelhecer@Saudável}

Considerando-se os métodos citados, principalmente Torrezzan [2009] e Passos [2011], e de aspectos específicos do público alvo pretendido (faixa etária, aspectos biológicos e 
fisiológicos, interesses, etc.), o Envelhecer@Saudável foi planejado seguindo as seguintes etapas:

* Etapa 1: organização da equipe de trabalho e agrupamento das informações (assuntos) iniciais que deveriam constar no material.

* Etapa 2: planejamento dos subsídios teóricos acerca do conteúdo a ser inserido ou produzido, além da escolha do nome.

* Etapa 3: escolha de formatos do MED - modelo de site: apresentação, conteúdos textuais e audiovisuais, com possibilidade de direcionamento para webquests ${ }^{1}$ específicas sobre cada assunto (tal recurso foi denominado no material de "Pesquisa Orientada", para melhor entendimento dos usuários); página em rede social (Facebook), a fim de proporcionar maior interação entre os participantes.

* Etapa 4: inserção dos recursos disponíveis, conteúdos e possibilidades para que o usuário possa explorar e navegar pelo site, webquests e rede social. Esta etapa foi pensada para auxiliar o usuário a explorar/conhecer as ferramentas propostas.

Respaldando-se nessas etapas, foi possível planejar e implementar o material cujo objetivo é propiciar a melhoria da qualidade de vida da população de mais idade, uma vez que seu conteúdo está voltado a atender as suas necessidades e limitações, e visa ainda possibilitar a inclusão digital (e social) dos seus usuários. Levando em conta os fatores do designer pedagógico [Torrezzan, 2009], o Envelhecer@Saudável foi desenvolvido a partir de metas gráficas, técnicas e pedagógicas.

No que diz respeito às metas gráficas, procurou-se utilizar recursos de design harmonizados à proposta pedagógica, a fim de estabelecer uma relação entre o cenário do ambiente virtual de ensino e aprendizagem (AVEA) e aspectos de layout adequados à faixa etária de uma população de mais idade [Barbosa et al., 2015]. A página de abertura do site (http://envelhecer-saudavel.wix.com/envelhecersaudavel) procura proporcionar uma interface atraente, com mensagem de boas-vindas e breve explicação sobre a navegação (não-linear). Procurou-se colocar todas as informações visíveis na tela, com cores e tamanho de fontes que permitissem uma melhor visualização. Além disso, os assuntos elencados para esse piloto e que foram considerados de maior relevância para serem tratados inicialmente, tais como Memória, Alimentação, Exercícios e Higiene, são facilmente encontrados pelos seus botões de acesso (Figura 1).

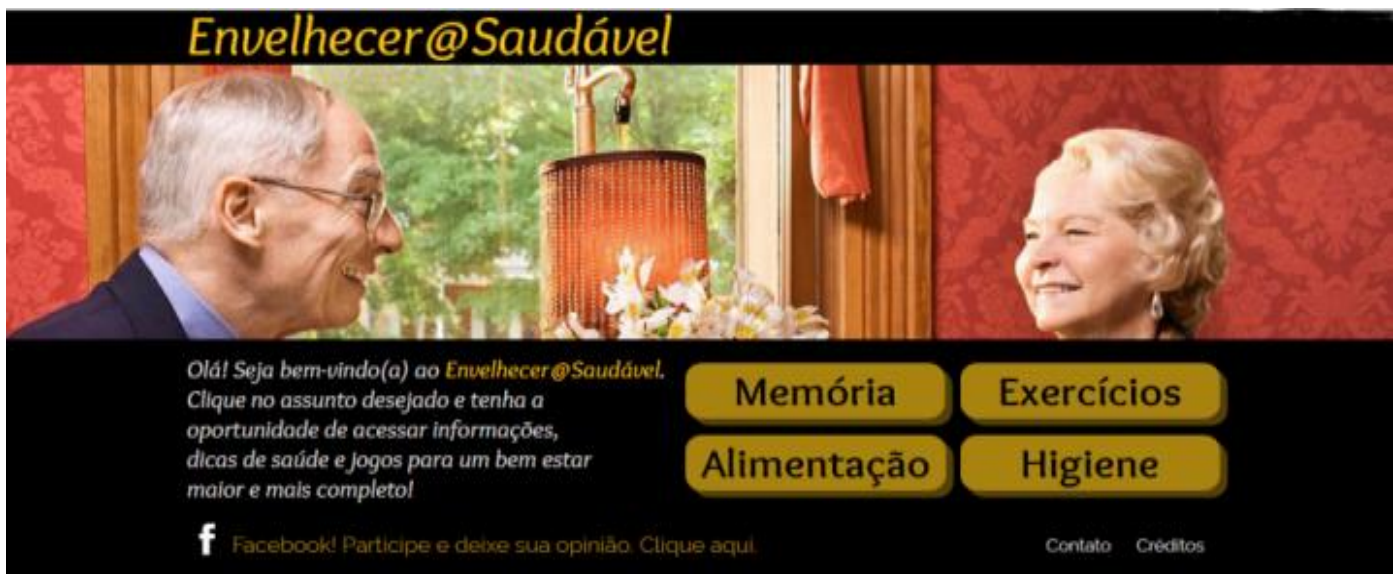

Figura 1: Interface de abertura do site Envelhecer@Saudável

${ }^{1}$ http://webeduc.mec.gov.br/webquest/ 
A apresentação visual de cada assunto após selecionado (Figura 2), segue um mesmo padrão de formatação, com um texto explicativo e botões de acesso para Vídeos, Leituras e Pesquisa Orientada, além das opções de ir para outro assunto desejado (ou voltar à página inicial) pela barra superior apresentada na tela, bem como a possibilidade de acessar a Biblioteca (com as referências dos materiais utilizados), ou sair do MED, sendo direcionado a página inicial do Google.

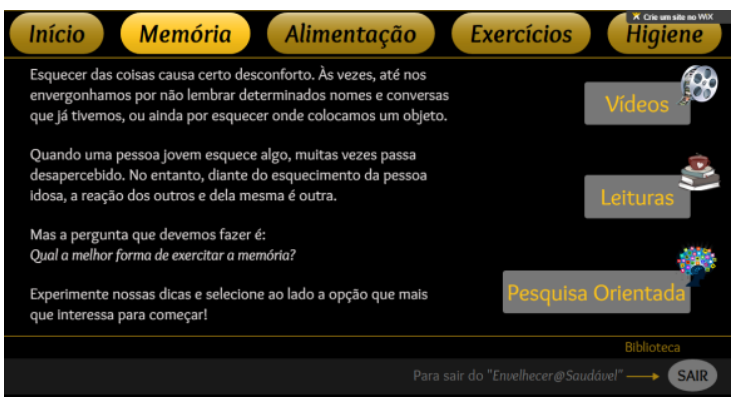

(a)

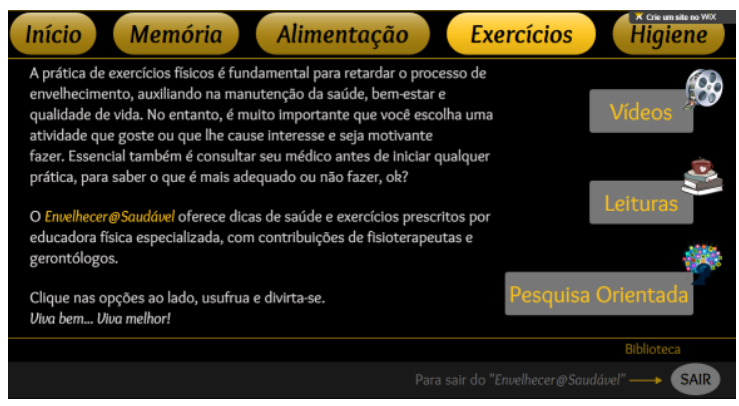

(b)

Figura 2: (a) Interface para o assunto "Memória"; (b) Interface para o assunto "Exercícios"

Vale salientar que uma das preocupações durante a escolha dos recursos e inserção dos conteúdos foi a de proporcionar ao usuário total visualização de qualquer um dos elementos dentro do próprio MED, sem necessidade de ser direcionado à uma página externa e com possível dificuldade para voltar ao material digital proposto. Como exemplos, o jogo da "Pirâmide dos Alimentos" e o "Cálculo do IMC (Índice de Massa Corporal)", sugeridos como uma das tarefas da "Pesquisa Orientada" para os assuntos Alimentação e Exercícios, respectivamente (Figura 3).

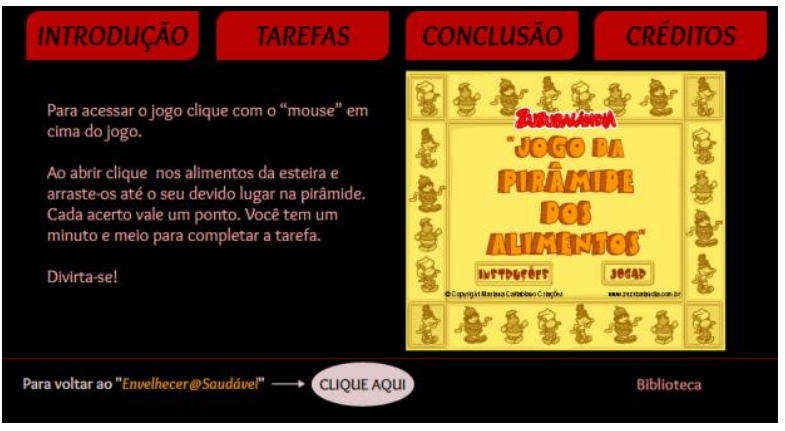

(a)

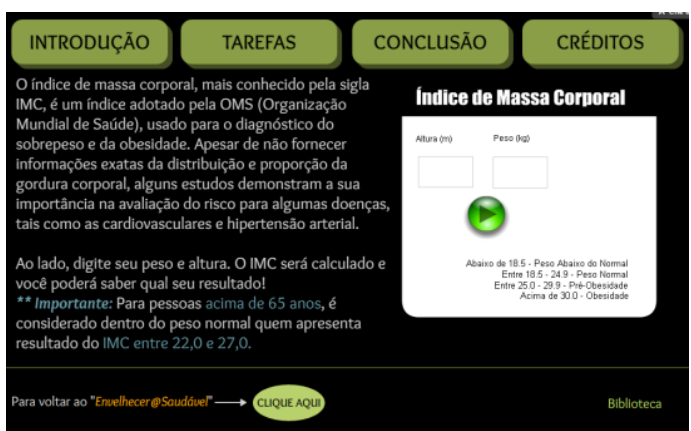

(b)

Figura 3: (a) Jogo "Pirâmide dos Alimentos"; (b) Tarefa "Cálculo do IMC"

Em relação às metas técnicas, levaram-se em consideração aspectos como usabilidade e acessibilidade, com estilos de navegação facilitados e, ao mesmo tempo, criativos. Sendo assim, além da criação do site na plataforma wix.com (http://pt.wix.com/), foram considerados aspectos como criar página na rede social Facebook com o intuito de possibilitar um MED mais acessível, interativo, colaborativo e com sequência não-linear, que fomente o interesse e a participação dos seus usuários. A página construída para a rede social, que pode ser acessada pelo link https://www.facebook.com/idosaudavel? fref=ts, visa possibilitar ainda ler informativos, bem como a participação em discussões com outros membros na própria página, ou em eventos que possam ser sugeridos. 
Nas metas pedagógicas, propiciaram-se situações de aprendizagem em que o usuário fosse capaz de atuar de forma mais investigativa e autônoma, por meio de vários recursos digitais, entre eles, imagens, vídeos, textos e atividades mais orientadas. Os assuntos eletivos, direcionados à faixa etária proposta a ser atendida pelo MED, foram disponibilizados inicialmente por meio do site e, posteriormente, inseridos na rede social para reflexão e discussão. No caso das atividades mais específicas, tem-se a Figura 5, que apresentam exemplos de páginas da "Pesquisa Orientada" (direcionada para um formato de webquest), do assunto Memória.

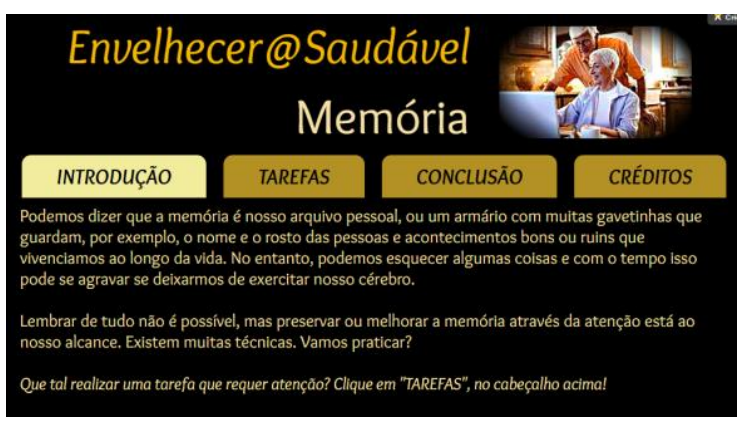

(a)

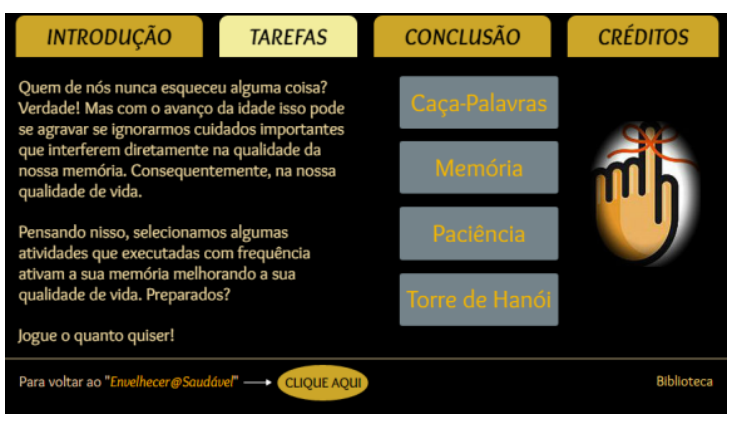

(b)

Figura 4: (a) Interface Inicial da "Pesquisa Orientada"; (b) Interface para Tela "Tarefas"

Por fim, salienta-se que o intuito de desenvolver um MED utilizando recursos variados é uma estratégia que visa estimular a capacidade do idoso para viver de forma autônoma e participativa socialmente por meio da interação e colaboração entre os seus pares. A próxima seção apresenta as considerações finais desta pesquisa e a proposta para trabalhos futuros a serem desenvolvidos.

\section{Considerações finais}

Pensar na construção de novas possibilidades para a educação de idosos utilizando materiais educacionais digitais exige repensar preconceitos ainda existentes de que essas pessoas não precisam ou não conseguem aprender por meio dessas ferramentas. Certamente, a inclusão digital na terceira idade depende da vontade e do interesse dos idosos. Porém, é imprescindível contar com o conhecimento de quem projeta e desenvolve tais materiais, além da colaboração (paciência e interesse) de quem ensina, adaptando o modo de ensinar e aprender como melhor convir para o idoso. Com base no referencial teórico, foi possível identificar que para criar um Material Educacional Digital é necessária uma metodologia que contemple um bom planejamento gráfico, técnico e pedagógico, propiciando a elaboração de um material interativo que, além de atrativo esteticamente, seja bem direcionado aos seus usuários e instigue a curiosidade dos mesmos, o que pode vir a contribuir de diferentes formas, abrindo novas possibilidades de ensino e aprendizagem para todos os envolvidos.

Como trabalhos futuros deste estudo, estão sendo realizadas uma validação da usabilidade da interface e uma avaliação do piloto desse MED junto ao público-alvo, a fim de adequá-lo cada vez mais aos objetivos pedagógicos e de interesse do adulto longevo. 


\section{Referências}

AMANTE, L.; MORGADO, L. Metodologia de concepção e desenvolvimento das aplicações educativas: o caso dos materiais hipermedia. In: Discursos, III Séries, $\mathrm{n}^{\circ}$ especial, pp. 125-138, Universidade Aberta , 2001.

BARBOSA, M. L. K.; ROESLER, V.; REATEGUI, E. "Um sistema de gerência e educação na saúde de idosos". 1. ed. Novas Edições Acadêmicas, 2015. v. 1. 132 p.

DOLL, J; CACHIONI, M; MACHADO, L.R. O idoso e as novas tecnologias. In: PY, L; FREITAS. Tratado de geriatria e gerontologia. São Paulo: Guanabara Koogan, 2016.

FONSECA, A. L. C. et al. "Construção do objeto de aprendizagem DIMOS -

Dispositivos Móveis e Seniors: uso e aplicação". 2015. Disponível em:

<http://www.lume.ufrgs.br/handle/10183/133423?locale-attribute=en>. Acesso em outubro de 2016.

GRANDE, T.P.F. "INSTRUMED: "Um instrumento para materiais educacionais digitais em dispositivos móveis para idosos". 2016. Dissertação apresentada ao programa de Pós-Graduação em Educação da Faculdade de Educação da Universidade Federal do Rio Grande do Sul. UFRGS.

KACHAR, V. "Terceira Idade \& Informática: Aprender revelando potencialidades". São Paulo: Cortez, 2003.

KACHAR, V. "Envelhecimento e perspetivas de inclusão digital". Revista Kairós Gerontologia, 13(2), INSS 2176-901X, São Paulo, novembro/2010: 131-147. Disponível em: <http://revistas.pucsp.br/index.php/kairos/article/view/5371>. Acesso em julho de 2016.

MACEDO, M. K. B.; PEREIRA, A. T. C. "Desenvolvimento de Recomendações de Acessibilidade e Usabilidade para Ambientes Virtuais de Aprendizagem Voltados para o Usuário Idoso". RENOTE. v. 7, n 1, julho, 2009. Disponível em: < http://www.seer.ufrgs.br/renote/article/viewFile/14035/7927>. Acesso em julho de 2016.

MACHADO, L. R.; BEHAR, P. A. "Construção de um objeto de aprendizagem voltado para pessoas idosas”. In: J. Sánchez (Ed.): Congresso Iberoamericano de Informática Educativa, Volume 1, pp 603-608, Santiago de Chile. Disponível em:

$<$ http://www.tise.cl/volumen6/TISE2010/Documento85.pdf $>$. Acesso em outubro de 2016.

MACHADO, L. R.; BEHAR, P.A. "Educação a Distância e Cybersêniors: um foco nas estratégias pedagógicas". Educação \& Realidade, Porto Alegre, v. 40, n. 1, p. 129148, jan./mar. 2015". Disponível em:

<http://www.seer.ufrgs.br/index.php/educacaoerealidade/article/view/45563/32233>. Acesso em julho de 2016.

ZIMMERMAN, F.; MOZZAQUATRO, P. M.; CUNHA, D.P. “A tecnologia Como Instrumento De Capacitação A Terceira Idade”. Disponível em:

$<$ http://www.unicruz.edu.br/seminario/anais/2013/CCAET/CIENCIAS\%20DA\%20C OMPUTACAO/C.ORAL/A\%20TECNOLOGIA\%20COMO\%20INSTRUMENTO\% 20DE $\% 20$ CAPACITA $\%$ C7\%C3O\%20A\%20TERCEIRA\%20IDAD.pdf $>$. Acesso em julho de 2016. 
NUNES, S. S. A acessibilidade na Internet no contexto da sociedade da informação. Dissertação de mestrado em Gestão de Informação. Porto, Universidade do Porto/Faculdade de Engenharia, FEUP, 2002.

PASSERINO, L. M.; PASQUALOTI, P. R. “A Inclusão Digital como Prática Social: uma visão sócio histórica da apropriação tecnológica em idosos". In: Portella, M, Gaglietti, M. and Pasqualotti, A. (org). Envelhecimento Humano: saberes e fazeres. Passo Fundo: Editora UPF, 2006. Disponível em: <http://www.brie.org/pub/index.php/sbie/article/view/466/452>. Acesso em julho de 2016.

PASSOS, P.C. S. J. "Interad: uma metodologia para design de interface de materiais educacionais digitais". Dissertação apresentada ao programa de Pós Graduação em Educação da Faculdade de Educação da Universidade Federal do Rio Grande do Sul. UFRGS. Disponível em: <http://www.lume.ufrgs.br/handle/10183/32234>. Acesso em julho de 2016.

PORTAL BRASIL. "Expectativa de vida aumenta em todo o mundo". Disponível em: < http://www.brasil.gov.br/saude/2014/12/expectativa-de-vida-aumenta-em-todo-omundo>. Acesso em agosto de 2016.

RODRIGUES, V.D.; PEREIRA, G.N. "O Idoso e sua Adequação à Qualidade de Vida na Sociedade Contemporânea”. Revista Acadêmica FEOL, 2016 - 177.8.219.7. Disponível em: <http://177.8.219.7:8081/revista/index.php/R1/article/view/57>. Acesso em julho de 2016.

SALES, M.B. "Modelo multiplicador utilizando a aprendizagem por pares focado no idoso". Florianópolis, 2007. 138 f. Tese (Doutorado) - Universidade Federal de Santa Catarina, Centro Tecnológico. Programa de Pós-graduação em Engenharia e Gestão do Conhecimento.

TAVARES, M.M.K.; SOUZA, S.T.C. "Os idosos e as barreiras de acesso às novas tecnologias da informação e comunicação". CINTED-UFRGS, Novas Tecnolgias na Educação. V. 10 No $^{\mathrm{o}}$, julho, 2012. Disponível em:< http://seer.ufrgs.br/renote/article/viewFile/30915/19244>. Acesso em julho de 2016.

TORREZZAN, C. A. W.; BEHAR, P. A. "Mapeamento de competências de equipes desenvolvedoras: um olhar na construção interdisciplinar de materiais educacionais digitais”. ETD - EducaçãoTemática Digital, Campinas, SP, v. 18, n. 1, p. 136-155, abr. 2016. ISSN 1676-2592. Disponível em: <http://periodicos.sbu.unicamp.br/ojs/index.php/etd/article/view/8638211>. Acesso em julho de 2016.

TORREZAN, C. A. W. "Design pedagógico: um olhar na construção de materiais educacionais digitais. 2009. 208 f. Dissertação (Mestrado em Educação) - Faculdade de Educação, Universidade Federal do Rio Grande do Sul, Porto Alegre, RS, 2009. Disponível em: <https://goo.gl/SWlSmZ>. Acesso em julho de 2016. 